\title{
Role of endocannabinoid system confirmed in patients with cirrhosis
}

The endocannabinoid system is upregulated in patients with cirrhosis, according to the results of a new study.

Endocannabinoids are involved in various central and peripheral physiological processes. "The endocannabinoid system has captured our attention, as a consistent amount of experimental data has shown that anandamide (AEA), the most important endocannabinoid, is implicated in the pathogenesis of the cardiovascular alterations of cirrhosis," says Mauro Bernardi, one of the authors of the study.

As data from human studies were limited, the authors measured circulating levels of the two major endocannabinoids (AEA and 2-arachidonoylglycerol) and two endocannabinoid-like molecules (oleoyl-ethanolamine [OEA] and palmitoyl-ethanolamine [PEA]) in the hepatic and peripheral veins of 18 patients with cirrhosis. They also measured the levels of these molecules in liver tissue.
Circulating levels of AEA, PEA and OEA were higher in patients with cirrhosis than in controls, which suggests that the encocannabinoid system is upregulated in cirrhosis. Levels of PEA and OAE were also increased in cirrhotic liver tissue. Surprisingly, however, no correlations were found between endocannabinoid levels and hemodynamic data.

"Having performed research activity at the bedside, we are planning to return to the bench," concludes Bernardi. "Our main aim is to study the role of PEA and OAE, and better clarify that of AEA, in the pathophysiology of hemodynamic alterations of decompensated cirrhosis."

Isobel Franks

Original article Caraceni, P. et al. Circulating and hepatic endocannabinoids and endocannabinoid-related molecules in patients with cirrhosis. Liver Int. 6, 816-825 (2010) 